Article

\title{
Enriching Elementary School Mathematical Learning with the Steepest Descent Algorithm
}

\author{
Roberto Araya 1,*
}

Citation: Araya, R.. Enriching Elementary School Mathematical Learning with the Steepest Descent Algorithm. Mathematics 2021, 9, x. https://doi.org/10.3390/xxxxx
1 Centro de Investigación Avanzada en Educación, Instituto de Educación, Universidad de Chile, Santiago 8320000, Chile; roberto.araya.schulz@gmail.com

* Correspondence: roberto.araya.schulz@gmail.com;

\begin{abstract}
The Steepest Descent (or Ascent) algorithm is one of the most widely used algorithms in Science, Technology, Engineering, and Mathematics (STEM). However, this powerful mathematical tool is neither taught nor even mentioned in K12 education. We study whether it is feasible for elementary school students to learn this algorithm, while also aligning with the standard school curriculum. We also look at whether it can be used to create enriching activities connected to children's real-life experiences, thus enhancing the integration of STEM and fostering Computational Thinking. To address these questions, we conducted an empirical study in two phases. In the first phase, we tested the feasibility with teachers. In a face-to-face professional development workshop with 457 mathematics teachers actively participating using an online platform, we found that after a 10-minute introduction they could successfully apply the algorithm and use it in a couple of models. They were also able to complete two complex and novel tasks: selecting models and adjusting the parameters of a model that uses the steepest descent algorithm. In a second phase, we tested the feasibility with 90 fourth graders from 3 low Socioeconomic Status (SES) schools. Using the same introduction and posing the same questions, we found that they were able to understand the algorithm and successfully complete the tasks on the online platform. Additionally, we found that close to $75 \%$ of the students completed the two complex modeling tasks and performed similarly to the teachers.
\end{abstract}

Keywords: Elementary Mathematics; STEM; Mathematical Modeling; Computational Thinking; Steepest Descent Algorithm

\section{Introduction}

A large part of elementary school education is dedicated to teaching mathematics. Together with learning to read and write, mathematics is one of the building blocks of elementary education. Numeracy and literacy therefore account for most of the time spent at school. In the case of mathematics, a large portion of this time is dedicated to learning about and mastering algorithms, such as the four operations and operations with fractions. Although arithmetic algorithms are simple and consist of only a few rules, learning them takes several years, with many students struggling to learn them properly [1, 2].

Much of the difficulty is due to what is known as evolutionary mismatch or the evolutionary trap $[3,4,5,6]$. Our brain is the product of a long process of evolution that has taken place over millions of years. In this time, it has evolved highly efficient mechanisms to learn the knowledge and skills required for the life of hunter-gatherers. These are the learning mechanisms of imitation, play, storytelling and teaching, which allow learning to walk, speak, collect food, hunt, cook, fight against other bands, and maintain a productive social life in our own small tribe. These types of knowledge and skills are called biologically primary [7]. However, due to the enormous advances in cultural evolution over the last 5,000 years, today we need students to learn completely new things, often of a 
totally different nature and some of them very counter-intuitive. Students also need to learn to live and socialize with thousands of strangers. They have to learn to read and write, something for which the brain is not adapted, requiring intensive and guided practice and leading to a huge transformation of different areas of the brain [8, 9]. We also need them to learn abstract concepts that are totally different from the ones acquired by their ancestors, such as positional notation, negative numbers, fractions, and algebra. These are considered biologically secondary knowledge [7]. For this reason, students need very well designed and tested lesson plans in order to guide them along proven learning trajectories. Connecting these abstract concepts with concrete elements of every day hunter-gatherer life can be a fruitful didactic strategy [10]. This includes connections between numbers and objects, or between numbers and positions. By doing so, adding and subtracting becomes grouping or ungrouping, or even a navigational activity. Another example is the connection between mathematical modeling and building a board game such as chess, where beads can be used to represent agents.

A great deal of experience has been accumulated over the last 600 years regarding how to teach these new abstract concepts and their respective algorithms. In the history of mathematics education, the teaching of algorithms plays a central role; particularly algorithms with numbers and practical applications. For example, the arithmetic textbook Liber Abaci written in 1202 by Fibonacci [11] presented algorithms for performing calculations without the aid of the abacus, using instead the then novel Hindu Arabic positional notation. This notation, which is the one we use today, represents the numbers and algorithms used with abacuses in a more abstract way. Later, in 1478, the Arithmetic of Treviso [12], the first printed arithmetic textbook, teaches arithmetic algorithms and how to apply them to business practice. It was a book with real-life applications, which was highly relevant to students and of great interest for the practical training of merchants. Filled with exercises based on business transactions, it set a precedent for what is perhaps still the most common type of exercise found in arithmetic textbooks.

However, new advances in science and technology require the introduction of new concepts and ways of Computational Thinking $[13,14,15]$. In recent centuries, the number of mathematical concepts and algorithms invented has grown exponentially. They are used in several practical and engineering problems. For example, linear programming algorithms, spectral methods, Kalman filters, neural networks, text mining algorithms, and many others are used in science and engineering. It is a dynamic process that continues to accelerate and put enormous pressure on an already overcrowded curriculum. However, these scientific and technological advances also make some algorithms almost obsolete. With a calculator or a smartphone we can calculate everything that is needed in commerce. Students therefore need not be very skillful in using an abacus or basic arithmetic algorithms for performing long divisions with big numbers. This situation frees up time to introduce new concepts and algorithms that are increasingly important in STEM and Computational Thinking.

Which new concepts and algorithms have been included recently on the curricula of different countries? One important case is Mathematical Modeling. Nowadays, it is considered one of the most important mathematical skills. This ability was included in the 2003 PISA Framework [16] and the Common Core State Standards for Mathematics in the USA. [17]. Developing and using models is also a basic practice in the Next Generation Science Standards (NGSS) [18], and delimiting and defining the system under study and making a model of it is one of the core crosscutting concepts for K12 Education in NGSS $[19,20]$. Building models is a basic thinking activity [21]. We are continuously imagining landscapes, situations, relations, and using them to run simulations in our heads in order to make decisions and act upon them. Most of these models are implicit. In mathematical modeling, we try to make them as explicit as possible, specifying their main components and the relations between them. For example, if you are planning to build a bridge, you start by imagining different possible bridges and the traffic across them. This is normally an implicit process that is done automatically. If you have more time and are in charge of 
designing the bridge, then you might try to imagine its components and how it will respond to traffic loads, water flow, and wind. You therefore start a more deliberate and explicit process. You can build a small toy bridge, test it, and measure its response to different stresses. You then have to think about how to scale it. Here it is critical that you try to determine the relations between the different components, testing them on your toy model and eventually on a more realistic model. This whole process, from an initial implicit model to an explicit model with mathematical specifications, is the mathematical model building process that allows us to make better decisions. It is a key ability that we need our students to learn and start using systematically in their daily lives.

Why Steepest Descent algorithm? Why with modeling? The Steepest Descent is one of the most widely used algorithms in applied Mathematics and STEM. In Mathematics it is at the core of Optimization and central to Dynamical Systems. It is critical in Computer Sciences for Machine Learning and Natural Language Processing. In Physics it is used to estimate trajectories with Scalar Potentials such as Gravitational and Electric Potential. In Chemistry and Biology, it is the core idea behind Chemotaxis and Tropisms. In Economics it is used in Decision Theory and Econometrics. It is also a common tool used in mathematical modeling and engineering. Several popular mathematical models are based on finding trajectories of particles or agents that optimize scalar potentials. This means that at each moment the next movement of the particle or agent is in the direction of steepest descent or steepest ascent. It is a powerful Computational Thinking tool.

Why, then, is it not used in K12 education? Steepest descent is normally introduced in Differential Calculus courses. This is because minus the gradient gives the direction of steepest descent when a linear tangent approximation to the scalar potential is used. However, in a discrete world, where the potential function is discrete or approximated using a discrete function, it can be formulated to move to the neighboring cell with the smallest (or highest) value. In this case, there is no need for differential calculus and the algorithm can be introduced in kindergarten or elementary school.

Our research question therefore asks whether it is feasible for elementary school students to learn the steepest descent algorithm and use it for modeling, while aligning with the standard school curriculum. If so, can it be used to create enriching activities connected to children's real-life experiences, enhance the integration of STEM and foster Computational Thinking?

\section{Materials and Methods}

We use four methodological strategies: applying a specialized framework for modeling, designing steepest descent activities with real-life models based on the interests of elementary school students, testing the activities during a professional development workshop for teachers, and testing the activities with fourth grade students.

The first methodological strategy is the use of a specialized framework for introducing mathematical modeling. This is the Use-Select-Adapt-Build (USAB) framework of Mathematical modeling $[22,23]$. This is a framework designed to introduce Mathematical Modeling skills in the K-12 school system. The strategy is to emulate how scientists, engineers, and other professionals do mathematical modeling. Some people have built models from scratch, but this is very uncommon. Normally, experts have been working for several years on existing types of models. We therefore need to know the main types of mathematical models that are used. There are also certain specific patterns and kinds of techniques that are used by experts. We need to identify these and then teach them to our teachers. Based on what the experts do, we have proposed a strategy for teaching mathematical modeling. This strategy consists of four stages [23]. The first stage is to make students USE certain types of typical and fertile [18] mathematical models; the student learns certain ways of thinking with models that can be used in a wide range of situations. Once the student knows how to use a model and has used it several times, then the second stage begins. During this stage, the student has to SELECT the most appropriate model from a set of two to five options. In the third stage, they have to ADJUST the parameters in a 
model in order to best fit a situation. In the fourth stage, they have to BUILD a new model or adapt an existing model for a new situation. The USAB framework is similar to other strategies [24].

It is essential that the models introduced are really fertile or generative. They must also be adaptable so as to include more details and complex phenomena in higher grades. In this way, every year students will review the same types of model but using more sophisticated mathematics and more parameters in order to account for more realistic situations. This strategy will ensure that they incorporate particular strategies and ways of thinking that can be used in building important and widely used types of mathematical and computational models.

The second methodological strategy is the design of steepest descent activities as a part of mathematical modeling. We designed four items using models of real-life experiences that could be of great interest to students: locomotion of bacteria $[25,26]$ and locomotion of dogs. The first item has 3 questions. The goal is to test the basic understanding of the scalar potential model and the steepest descent algorithm. However, in the example we use the steepest ascent version. In each question the user has to apply the Steepest Ascent algorithm to generate the trajectories of three bacteria as shown in Figure 1. In this item, the user has to Use the model. At each step, the user therefore has to consider the rules of an already precisely described model and the current position of the bacteria in order to work out its next position. This mental process must be repeated until the bacteria cannot continue ascending. Thus, the user has to manage several thinking process, all of which correspond to the Use component of the USAB framework.

\begin{tabular}{|c|c|c|c|c|c|c|c|c|c|c|c|}
\hline \multicolumn{12}{|c|}{$\begin{array}{l}\text { Mark the path that the bacteria must follow, which always moves to the neighboring cell where the } \\
\text { higher number is (the number represents the amount of nutrients), until it no longer finds a higher } \\
\text { number. }\end{array}$} \\
\hline \multirow{5}{*}{$\begin{array}{l}\text { Mark the path of the } \\
\text { bacteria that begins } \\
\text { above where the two } \\
\text { is located. }\end{array}$} & 2 & 1 & 3 & 5 & 6 & 3 & 3 & 4 & 3 & 0 & \multirow{5}{*}{$\begin{array}{l}\text { Mark the path of the } \\
\text { bacteria that begins } \\
\text { above where the zero is } \\
\text { located. }\end{array}$} \\
\hline & 10 & 2 & 2 & 2 & 7 & 4 & 5 & 5 & 4 & 2 & \\
\hline & 11 & 13 & 2 & 3 & 4 & 9 & 10 & 3 & 14 & 13 & \\
\hline & 21 & 3 & 19 & 4 & 4 & 19 & 5 & 14 & 4 & 3 & \\
\hline & 22 & 4 & 4 & 20 & 5 & 25 & 6 & 16 & 25 & 5 & \\
\hline \multirow{7}{*}{$\begin{array}{l}\text { Mark the path of the } \\
\text { bacteria that begins } \\
\text { above where the five } \\
\text { is located. }\end{array}$} & 30 & 24 & 5 & 18 & 6 & 25 & 6 & 6 & 19 & 4 & \\
\hline & 32 & 4 & 6 & 16 & 6 & 17 & 6 & 6 & 4 & 4 & \\
\hline & 2 & 4 & 26 & 15 & 12 & 19 & 27 & 35 & 25 & 15 & \\
\hline & 32 & 34 & 36 & 37 & 38 & 10 & 47 & 41 & 35 & 14 & \\
\hline & 38 & 44 & 35 & 8 & 39 & 28 & 29 & 38 & 18 & 13 & \\
\hline & 11 & 4 & 4 & 6 & 40 & 38 & 9 & 10 & 11 & 12 & \\
\hline & 0 & 5 & 3 & 4 & 41 & 8 & 8 & 5 & 4 & 2 & \\
\hline
\end{tabular}

Figure 1. Screenshot of Item 1. Note that this item has three questions. The user has to mark the path that the bacteria will follow, which always moves to the neighboring cell with the highest number (the number represents the amount of nutrients), until it no longer finds a higher number.

Figure 2 shows the solution. There are three trajectories. The user has to consecutively click on the right cell in order to generate the trajectories. 


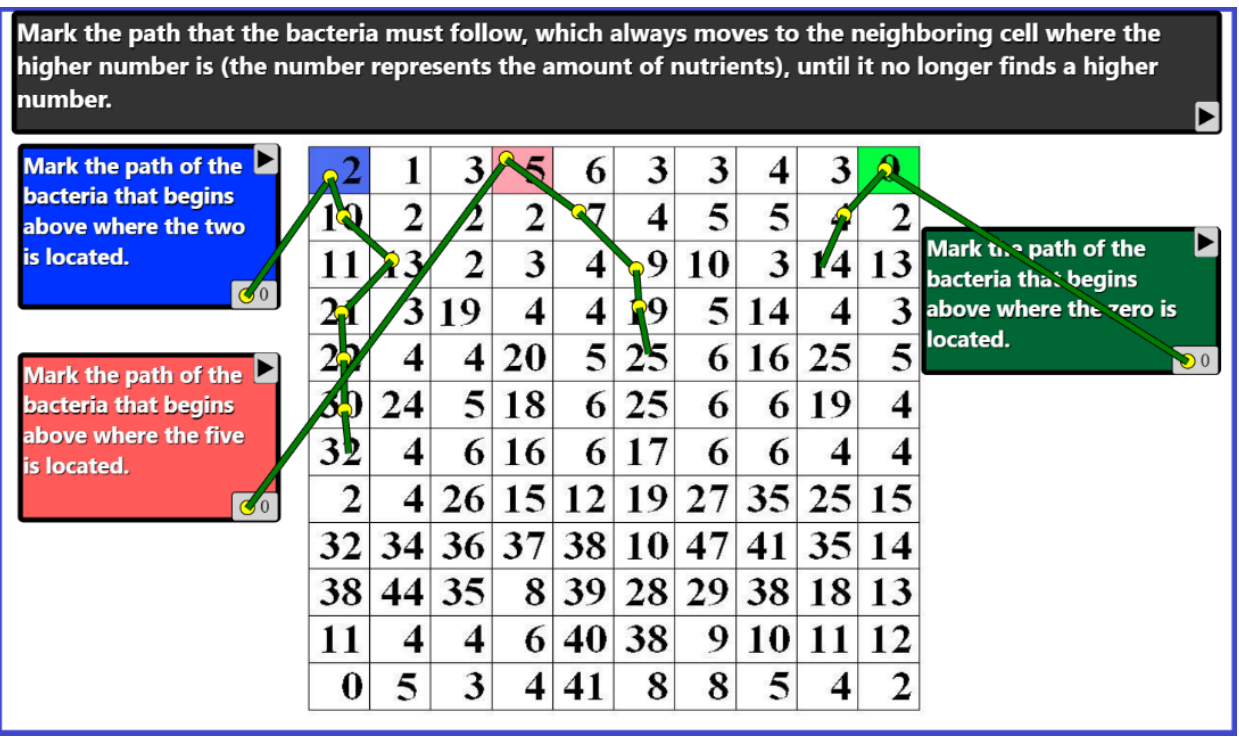

Figure 2. Correct answers to Item 1 . There are three trajectories, one for each question.

In the second item, the user again has to apply the Steepest Ascent algorithm. However, this time it is to generate the trajectory of a dog (Figure 3). This is a direct generalization of the previous model. It has just two changes. Firstly, there is a change in the agent. Instead of a bacteria, we have a dog. Secondly, there is a change in the meaning of the potential function. Instead of nutrients for bacteria, the scalar potential now represents the intensity of the smell. This again involves the Use component of the USAB modeling framework, with the student having to consider a small adaptation in how the numbers on the board are interpreted.

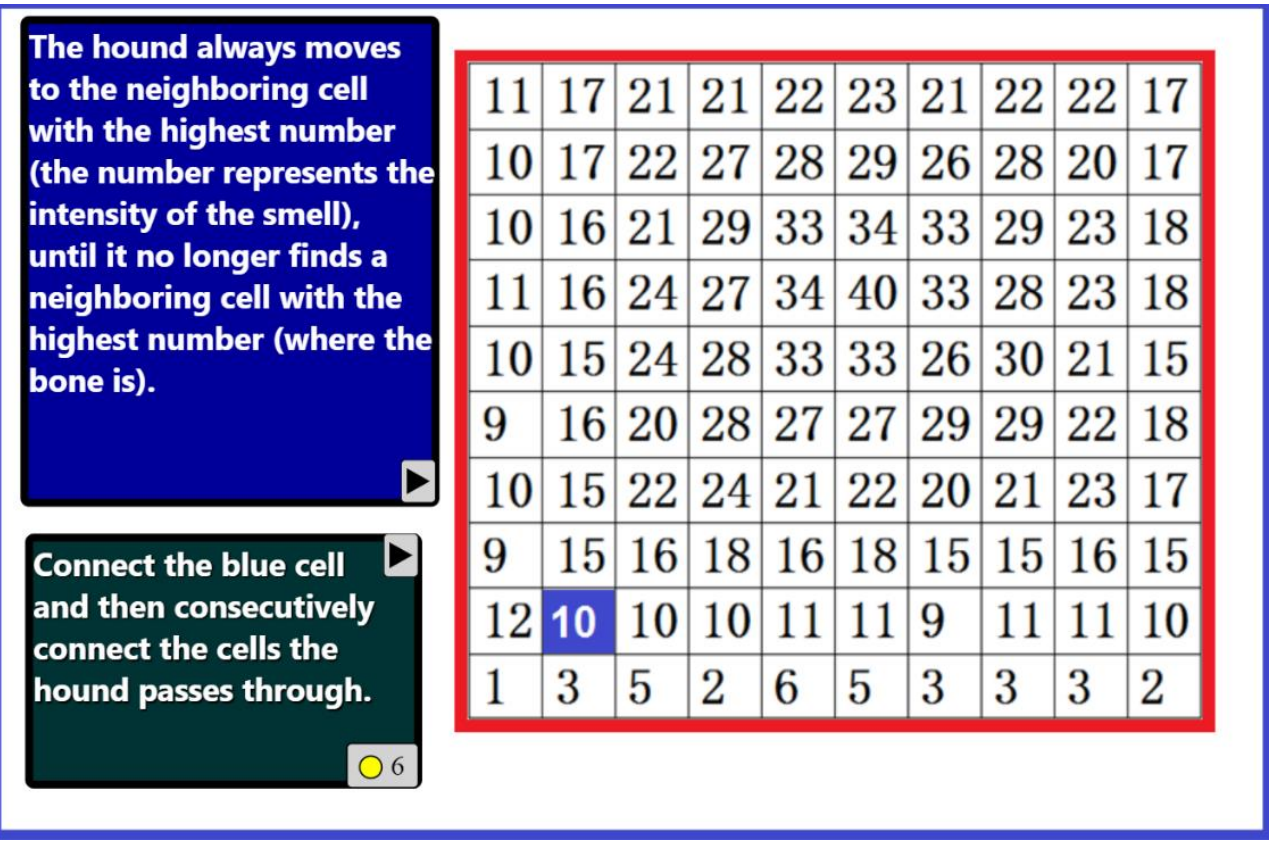

Figure 3. Screenshot of Item 2. Here, the user has to connect the blue cell and then consecutively connect the cells through which the dog passes, considering that it always moves to the neighboring cell with the highest number (the number represents the intensity of the smell), until it no longer finds a neighboring cell with the highest number (i.e. where the bone is).

Figure 4 shows the corresponding solution. The user has to consecutively click on the right cell in order to generate the correct trajectory. 


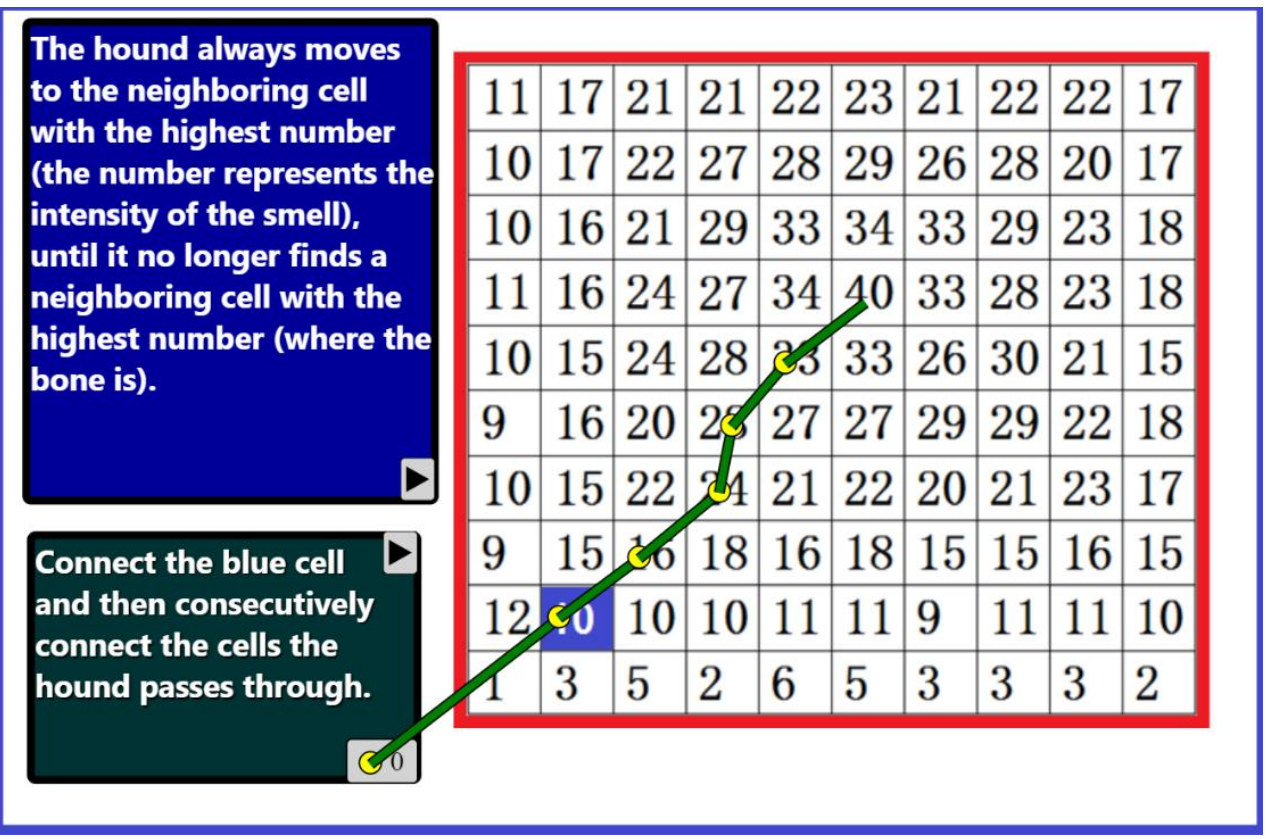

Figure 4. Correct answer to Item 2.

The third item asks the user to select a model (Figure 5). This represents a higherorder thinking skill. The user has to mentally simulate the trajectories for both models. Then, at some point, the user has to realize a new interpretation of the problem. During the thinking process the user has to discover that the problem translates to the following: on which board are there two local maximums? This item corresponds to the Select stage of the USAB framework.

\begin{tabular}{|c|c|c|c|c|c|c|c|c|c|c|c|c|c|c|}
\hline \multicolumn{15}{|c|}{$\begin{array}{l}\text { The hound always moves to the neighboring cell with the highest number (the } \\
\text { number represents the intensity of the smell), until it no longer finds a } \\
\text { neighboring cell with the highest number (where the bone is). }\end{array}$} \\
\hline \multicolumn{15}{|c|}{$\begin{array}{l}\text { Connect the board that represents a courtyard where two bones are hidden in } \\
\text { different places. }\end{array}$} \\
\hline 19 & 26 & 24 & 23 & \begin{tabular}{|l|}
22 \\
\end{tabular} & 15 & 8 & 14 & 14 & 11 & 14 & 10 & 14 & 13 & \\
\hline 20 & 25 & 32 & 26 & 19 & 15 & 8 & \begin{tabular}{|l|}
19 \\
\end{tabular} & 20 & 19 & 13 & 10 & 19 & 20 & \\
\hline 21 & 27 & 27 & 25 & 20 & 16 & 8 & 22 & 26 & 15 & 15 & 14 & 21 & 14 & \\
\hline 21 & 19 & 19 & 22 & 18 & 13 & 9 & 18 & 21 & 13 & 21 & 22 & 21 & 22 & \\
\hline 14 & 14 & 15 & 14 & 14 & 14 & 9 & 14 & 14 & 15 & 21 & 25 & 25 & 26 & \\
\hline 8 & 9 & 8 & 8 & 6 & 10 & 9 & 7 & 10 & 15 & 20 & 25 & 33 & 24 & \\
\hline 3 & 3 & 3 & 1 & 1 & 1 & 2 & 1 & 6 & 14 & 19 & 25 & 26 & 27 & \\
\hline
\end{tabular}

Figure 5. Screenshot of Item 3 . In this item the user has to consider the same rule of motion as in Item 2 and then connect the board that represents a courtyard where two bones are hidden in different places. 
The fourth item asks the user to adjust the parameters of a model (Figure 6). This item also requires higher-order thinking skills, where several options have to be tested. Here, the user has to realize that the problem can again be interpreted as having to find local maximums. Once they discover that the board has two local maximums, the user has to change the number in one of them in order to make sure that all trajectories will lead to the desired location. This item corresponds to the Adjust component of the USAB framework.

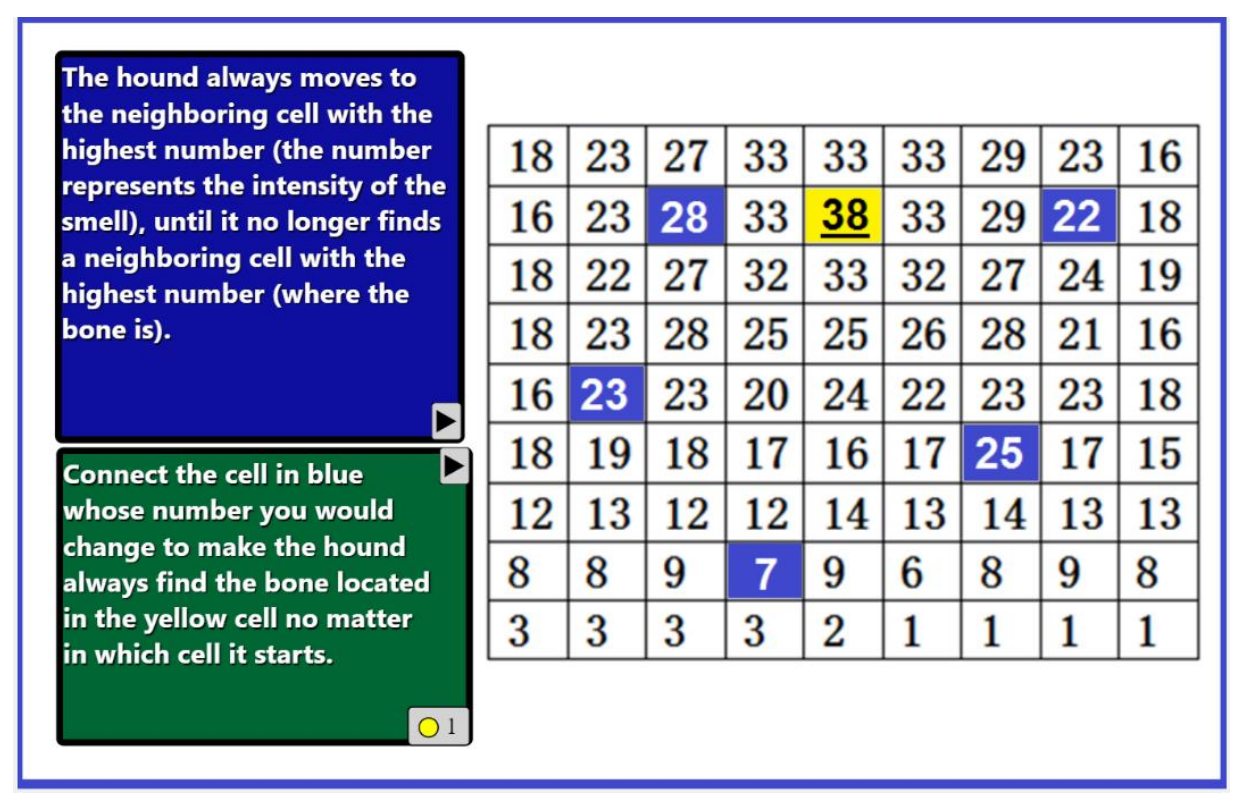

Figure 6. Screenshot of Item 4 . Here the user has to consider the same rules of motion as in Items 2 and 3 , and then connect the cell in blue whose number should be changed in order for the dog to always find the bone located at the yellow cell no matter where it starts.

In all 4 of the items, the numbers in the cells are all natural numbers below 50 . The items require the user to do a series of number comparisons. This is a typical learning objective on the elementary mathematics curriculum. For fourth graders, the numbers could be larger than 50, or include fractions, decimals, or additions and subtractions of integers. This means that the exercises can be adapted to include more complex numbers and expressions based on the grade level and learning objectives. Nevertheless, for the present study, all numbers are integers between zero and 50 .

The four items measure some of the learning objectives that are typically found in the number strand of the curriculum, as well as the students' mathematical modeling skills. Furthermore, they also introduce the user to computational thinking.

The third methodological strategy is to test the idea with teachers. To do so, we designed a Mathematical Modeling seminar for teachers that included the steepest descent algorithm. The seminar focused on why we should teach mathematical modeling, explaining that it has been included on the international PISA test since 2003, as well as on the TIMMS test for $4^{\text {th }}$ and $8^{\text {th }}$ grade. The seminar provided a historical overview of Mathematical Modeling, presenting Archimedes' Method [27] and ancient Egyptian board games [28]. The seminar then focused on what has changed with the increasing use of computers and computer simulations. It argued that mathematical models are enhanced by these technologies, leading to a huge increase in the ability to predict what will happen in different scenarios and therefore improve decision-making. Thus, traditional mathematical skills must be complemented with the knowledge that is needed to detect patterns, as well as to select and adjust mathematical models. The seminar included a 90-minute workshop during which teachers were introduced to several models. The workshop included a 10-minute introduction to the steepest descent algorithm used in said models. At the end of the workshop, we asked the participants to answer the previous four items on 
modeling using their personal devices (smartphone or tablet). Teachers were shown how to login to the ConectaIdeas platform $[29,30]$ and how to generate a trajectory. During the last 15 minutes of the workshop, the teachers connected their devices to the wifi, logged in to the ConectaIdeas platform, and answered the four items.

The fourth methodological strategy was testing the same activities with elementary school students. Ninety fourth graders (51 boys and 39 girls) from three different schools participated in a 90-minute session on mathematical modeling and the steepest descent algorithm. The students were given the same 10-minute introduction that was delivered during the teacher workshop. The students then had to answer the same four items using the ConectaIdeas platform. We studied whether they were able to understand the steepest descent algorithm and use it to understand modeling with scalar potentials, as well as looking at the extent to which they were able to solve the more complex items.

\section{Results}

The face-to-face seminar attracted almost nine hundred teachers. Most of them also attended the mathematical modeling workshop. The workshop was well evaluated by the teachers. They appreciated the modeling exercises for different grade levels and with practical examples. They also valued the association of mathematics with experiences and areas that they had never thought could be related to mathematics. However, the biggest concerns were how to evaluate this new modeling skill.

Not all of the teachers brought a device that could connect to internet and the ConectaIdeas platform. We received responses from 457 teachers: 175 males and 282 females. Virtually all of the teachers who answered at least one item went on to complete all four (Table 1). However, there may have been teachers who had suitable devices but chose not to respond.

In the case of students, we recruited three schools, with a total of 90 fourth grade students (51 boys and 39 girls). The majority of students answered all of the items, with the exception of Item 1 (Table 1). Only $42.2 \%$ of the students answered Item 1 . However, $95.6 \%$ of the students answered Item 2 , which is very similar to Item 1 and has the same level of difficulty. Item 1 was a basic item used mainly to check understanding and how to use the platform.

Table 1. Percentage of teachers and students who answered each item.

\begin{tabular}{lrrrrr}
\hline Subjects & $\mathbf{N}$ & \% Item 1 & \% Item 2 & \% Item 3 & \% Item 4 \\
\hline Teachers & 457 & 99.6 & 99.3 & 98.9 & 98.9 \\
Students & 90 & 42.2 & 95.6 & 76.7 & 72.2 \\
\hline
\end{tabular}

The teachers and students' performance was well above chance (Table 2). The first two scores are well above random selection. For Item 1, each of the three questions has many possible answers. Even if only empty neighboring cells are considered, there are eight options for the first move alone. Therefore, the probability of randomly choosing the correct option is just $1 / 8$. This is followed by the second move, where the probability of randomly choosing the correct option is again $1 / 8$. The probability of randomly getting the 2 moves right is $(1 / 8)^{2}$. With three movements, the probability decreases to $(1 / 8)^{3}$. The probability of randomly getting all three questions right in 3 moves each is $(1 / 8)^{3}(1 / 8)^{3}(1 / 8)^{3}=0.000000008$. This means that choosing neighboring cells at random gives an expected score of 0.0000008 points. The real expected score is even lower since some trajectories are longer than 3 moves. Thus, in practical terms, the probability of answering correctly by chance is zero. Similarly, for Item 2 the expected score is 0.19 points when choosing at random. For Item 3, selection by chance gives 50 points. This means that the teachers and students in our study scored above chance with a 95\% confidence level. The closest to chance is Item 3. However, the scores minus the Confidence Interval (CI) width does not hit the chance score. Therefore, the score is above chance with a 95\% confidence level. Interestingly, the students performed significantly better than the teachers 
on Item 3 (Figure 7). However, we have to consider that only $76.7 \%$ of the students answered this item, compared with $98.9 \%$ of the teachers. Item 4 has 5 options. Therefore, the expected score is 20 points when choosing at random.

Table 2. Average score by teachers and fourth grade students

\begin{tabular}{lrrrr}
\hline Subjects & Item 1 & Item $\mathbf{2}$ & Item $\mathbf{3}$ & Item 4 \\
\hline Teachers & 52.3 & 59.7 & 60.8 & 56.8 \\
Students & 43.2 & 49.7 & 77.5 & 50.1 \\
Chance & 0 & 0.19 & 50 & 20 \\
\hline
\end{tabular}

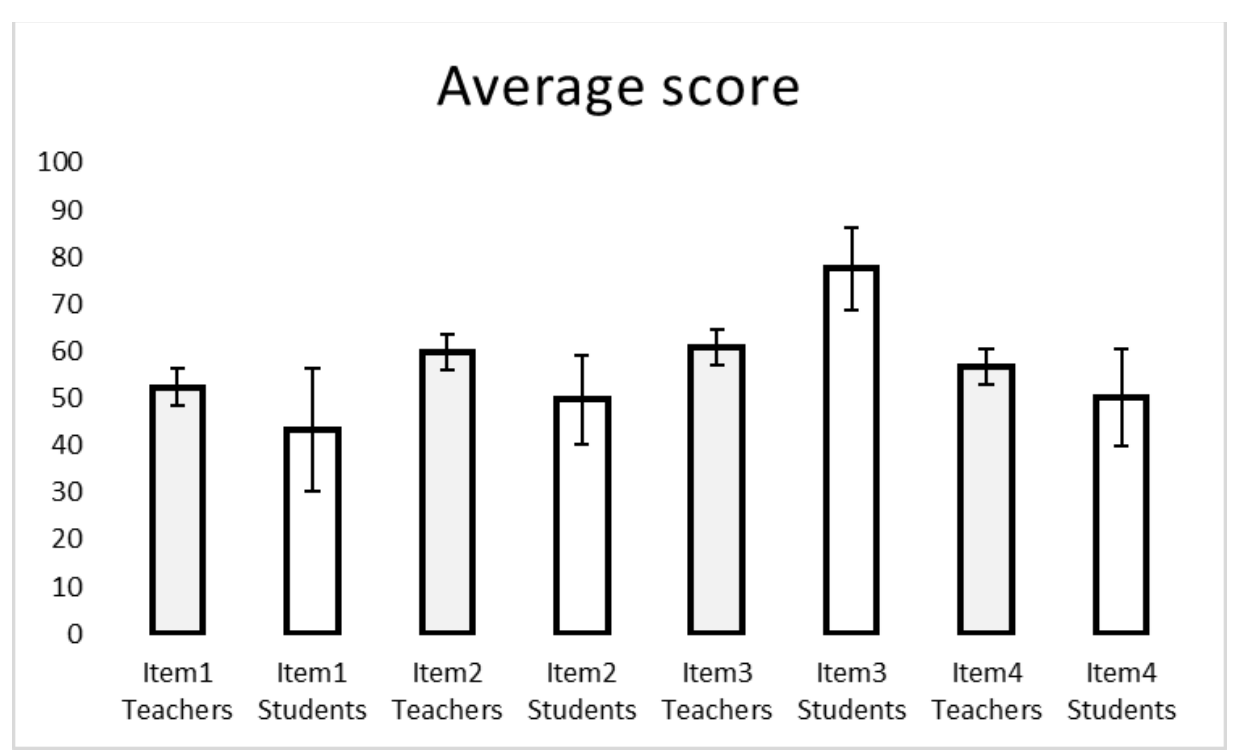

Figure 7. Average score by teachers and students for each item, with $95 \%$ Confidence Intervals.

The response times for the teachers was almost twice that of the students (Table 3). This is because the teacher data includes a number of very long response times. Some teachers opened the question during the workshop and answered them once the workshop had finished. In the case of the students, they all responded during the session. Item 1 takes a longer time to answer because it has three questions, each of them requiring a separate trajectory. Item 3 and 4 take less time to answer because they do not require a trajectory.

Table 3. Average response times (in seconds) for each item.

\begin{tabular}{lrrrr}
\hline Subjects & Item 1 & Item 2 & Item 3 & Item 4 \\
\hline Teachers & 730 & 537 & 204 & 140 \\
Students & 478 & 219 & 78 & 79 \\
\hline
\end{tabular}

\section{Discussion}

There are many algorithms that are taught in elementary mathematics. Most of them stem from previous advances in mathematics and technology, such as the abacus and the positional system. Nevertheless, today there are a number of new algorithms that are particularly relevant to more modern applications. The steepest descent (or ascent) algorithm is an extremely popular and useful algorithm in STEM and Computer Sciences. However, it is not taught in the K12 school system. Our research question therefore looked to determine whether this powerful algorithm and its use in mathematical modeling can be learned by elementary school students. In this study, we have implemented a practical 
experience of mathematical modeling with scalar potentials using this algorithm. Following a 10-minute introduction, we have found that teachers and fourth grade students were able to understand and apply the algorithm to a series of an increasingly complex models. The users started by directly applying the models, before moving on to selecting and adjusting said models. These are more complex and advanced modeling tasks that require less direct and more creative mathematical thinking.

Item 3 requires users to select a model that, when applying the steepest ascent algorithm, generates the desired behavior. This task was not similar to the ones used in the introduction. It was completely new to users. It requires them to mentally simulate the dog's behavior in both models and generate the corresponding trajectories. Furthermore, the user can also translate the problem to another problem: determining in which model there are two local maximums. $98.9 \%$ of the teachers answered this problem, with an average score of 60.8 points. In the case of the students, $76.7 \%$ of them answered the problem, with an average score of 77.5 points. In both cases, the scores suggest that the users understood the problem since they are statistically above chance. While the scores might also suggest that the students understood the problem better than the teachers, we have to consider that just over a quarter of the students failed to answer. Furthermore, practically all of the teachers answered said item.

Item 4 also proposes a completely new situation. It is not directly related to the initial training nor to the first two items. Here, the user is given five options on the board where they can modify the value in the respective cell. In each case, the user must think about the impact it will have on the dog's behavior and its corresponding trajectory, regardless of wherever it starts. This means mentally exploring a huge number of possible trajectories. Simulating them all is not possible given the capacity of the working memory of each user. Even if the subject is writing them down it would take a great deal of time. The way to solve the problem is to imagine that it boils down to finding a way to make sure the board has only one local maximum. This requires a leap in the conception of the problem. $98.9 \%$ of the teachers answered this item and scored an average of 56.8 points. $72.7 \%$ of the students answered the same item and managed an average score of 50.1 points. In both cases, the scores are far above chance. Furthermore, the students' average score is statistically equal to the teachers' average score. However, again it must be considered that just over a quarter of the students did not answer this item.

Items 3 and 4 test a computational way of thinking. Students must imagine that the world is modeled on a board or array with discrete cells, containing numbers that represent nutrients, and they must conceive the behavior of organisms as if they were automata that follow precise rules. The results show that in this computational representation they manage to think of changes in the environment as changes in the numbers of the cells. Therefore, students begin to model behavior in a fully computational way.

The activities were an enriching experience that modeled and applied the steepest descent algorithm to everyday problems that are of interest to the students. Children are naturally attracted to animals and their behavior. One activity is about the navigation of bacteria. Bacteria are a familiar topic for the students as they learn that millions of them live and move within their bodies; some helping with digestion, while others cause diseases. Another activity is about dogs and their sense of smell. This is undoubtedly the closest animal to children. Understanding how the dog moves when it looks for food using its sense of smell is an activity that is very close to the students' daily lives. In both cases, mathematics helps them understand phenomena that they experience every day, and that go beyond the arithmetic exercises typical of school textbooks that are largely circumscribed to transactions in commerce.

One critical challenge in STEM education is integration [31]. However, one of the most powerful thinking tools that the experts use for Mathematical Modeling across disciplines is the scalar potential. It is used in physics (gravity and electromagnetism), chemistry (chemotaxis) and biology (tropisms of microorganisms, bacterial chemotaxis) [32,33]. Here, we have shown that by using steepest descent algorithm, this tool can be taught to elementary school students integrating biology with mathematics. 
Additionally, the activities can be perfectly aligned to the requirements of the current curriculum, both for Mathematics and Science. They can be easily adapted to be taught using different media: inside the classroom on paper or whiteboards, outdoors in school yards, or on students' smartphones. Our results show that it is feasible to introduce the algorithm in elementary schools.

\section{Conclusions}

We conducted an experience with 475 teachers and then repeated exactly the same experience with 90 fourth-grade students. In both cases, the users answered 4 items containing a total of 7 questions. We found that in both cases there is an understanding of the models and the algorithm. Furthermore, on two completely new items for teachers and students, we found empirical evidence that suggests they were able to generalize and gain insight. This is a particularly encouraging finding. It suggests that these powerful STEM concepts and tools can be easily introduced into the school system. In other words, despite the potential difficulties foreseen by the evolutionary trap, students can quickly understand this algorithm in navigation problems. One possible explanation is that the navigation problem that organisms solve by using local information from their environment to move to where there are more nutrients or intensity of nutrient odors is, ultimately, a basic foraging problem that all animals and our ancestors have repeatedly solved for survival. In this sense, the steepest descent (or ascent) algorithm is equivalent to a navigation rule for which our brain is very well adapted.

Additionally, we introduced this algorithm in activities that mathematically model real-life situations that are of great interest to fourth graders. These are activities that promote the integration of STEM; in this case mathematics and biology. Students can do similar examples integrating mathematics with other sciences and engineering. Moreover, the steepest descent algorithm is a powerful example of Computational Thinking. It is a way of thinking and modeling the world with an important impact on a great variety of problems. It is also at the core of two pillars of Computational Thinking: Machine Learning and Computational Modeling [14,15].

We can therefore conclude that introducing the Steepest Descent (or Ascent) algorithm is both feasible in elementary education and attractive to students, while also allowing greater integration in STEM and successfully promoting Computational Thinking.

Funding: This work was supported by the Chilean National Agency for Research and Development (ANID), grant number ANID/ PIA/ Basal Funds for Centers of Excellence FB0003.

Acknowledgments: Support from ANID/PIA/Basal Funds for Centers of Excellence FB0003 is gratefully acknowledged.

Conflicts of Interest: The author declares no conflict of interest. The funder had no role in the design of the study; in the collection, analyses, or interpretation of data; in the writing of the manuscript, or in the decision to publish the results.

\section{References}

1. Valverde, G.; Marshall, J.; Sorto, A. Mathematics Learning in Latin America and the Caribbean. In Learning mathematics in the 21st century: adding technology to the equation, 1st ed.; Arias Ortiz, E., Cristia, J.; Cueto, S. IDB, U.S.A., 2020, pp. 97-139

2. OECD. PISA 2018 Results (Volume II): Where All Students Can Succeed. PISA OECD Publishing: Paris, 2019.

3. Mayr, E. Systematics and the Origin of Species, from the Viewpoint of a Zoologist. New York: Columbia University Press. USA, 1942; pp. 224.

4. Lloyd, E.; Wilson, D.; Sober, E. Evolutionary Mismatch and What to Do About It: A Basic Tutorial. Evolutionary Applications. 2011, 2-4.

5. Van Gugt, M.; Li, N. The Evolutionary Mismatch Hypothesis Applications of Social Psychology: How Social Psychology Can Contribute to the Solution of Real-World Problems 2020

6. Pani, L. Is there an evolutionary mismatch between the normal physiology of the human dopaminergic system and current environmental conditions in industrialized countries? Molecular Psychiatry, 2000, 5, 467-475 
7. Geary, D.: Educating the evolved mind: conceptual foundations for an evolutionary educational psychology. In: Carlson, J.S., Levin, J.R. (eds.) Psychological Perspectives on Contemporary Educational Issues. Information Age Publishing, Greenwich, U.S.A., 2007

8. Henrich, J.: The WEIRDest People in the World: How the West Became Psychologically Peculiar and Particularly Prosperous, Farrar, Straus and Giroux, 2020

9. Dehaene S. Evolution of human cortical circuits for reading and arithmetic: The "neuronal recycling" hypothesis . In S .Dehaene , J . R . Duhamel , M . Hauser , \& G . Rizzolatti ( Eds .), From monkey brain to human brain ( pp . 133 - 157 ). Cambridge, MA : MIT Press, 2005

10. Araya, R.; Calfucura, P., Jiménez, A.; Aguirre, C.; Palavicino, M.; Lacourly, N.; Soto-Andrade, J.; Dartnell, P. The Effect of Analogies on Learning to Solve Algebraic Equations. Pedagogies: An International Journal. Special Issue The teaching of Algebra. 2010, Volume 5, Issue 3.

11. Fibonacci. Liber Abaci https://en.wikipedia.org/wiki/Liber Abaci

12. Swetz, F. Capitalism \& Arithmetic. Open court, Illinois. U.S.A. 1987

13. Wing, J.M. Computational Thinking Benefits Society. 2014 Retrieved from http://socialissues. cs.toronto.edu/index.html\%3Fp=279.html

14. Denning, P. Computational Thinking in Science. American Scientist, 2017, Vol. 105, No. 1.

15. Araya, R., Isoda, M., \& González, O. A Framework for Computational Thinking in Preparation for Transitioning to a Super Smart Society. Journal of Southeast Asian Education, 2020, 1, 1-15.

16. OECD. PISA 2003 Assessment Framework: Mathematics, Reading, Science and Problem Solving Knowledge and Skills - Publications 2003

17. Common Core Standards for Mathematics http://www.corestandards.org/assets/CCSSI Math\%20Standards.pdf

18. Next Generation Science Standards https://www.nextgenscience.org/

19. Krajcik, J.; Merritt, J. Engaging Students in Scientific Practices: What does constructing and revising models look like in the science classroom? Science Scope, 2012, v35, 7, 6-8

20. Krajcik, J. The Next Generation Science Standards A Focus on Physical Science. Science Teacher. 2013, v80 3, 27-37

21. Epstein, J. Why model? Journal of Artificial Societies and Social Simulation 2008, vol. 11, no. 412

22. Araya, R. Improving Math Education in Chile: Standards, e-Tutoring and Multiplayer Games. Proceedings of the APEC Seminar on Best Practices and Innovations in the teaching and learning of Science and Math. Penang, Malasia 2004

23. Araya, R. Introducing Mathematical Modeling Skills in the Curriculum. In Proceedings of APEC Conference in Math Modeling in the Mathematics Curriculum: Some Best Practices in APEC Economies. Indonesia, 2012, 52-64 http://publications.apec.org/publication-detail.php?pub id $=1362$

24. Lingefjard, T. Mathematical Modelling in Teacher Education - Necessity or Unnecessarily. In Blum et Al, Modelling and Applications in Mathematics Education. Springer Verlag. 2007

25. Loeb, J. Forced movements, tropisms, and animal conduct. Philadelphia, Lippincott, 1918.

26. Itskovits, E.; Ruach, R.; Zaslaver, A. Concerted pulsatile and graded neural dynamics enables efficient chemotaxis in C. elegans Nature Communications. 2018, 9:2866

27. Archimedes. The Method. https://en.wikipedia.org/wiki/The Method of Mechanical Theorems

28. Thibault, D. ASEB: The Game of Twenty Squares. An Ancient Egyptian Two-Player Board Game. https://cdn.myminifactory.com/assets/object-assets/59e48c87ac796/threedfiles/aseb.pdf

29. Araya, R.; Diaz, K. Implementing Government Elementary Math Exercises Online: Positive Effects Found in RCT under Social Turmoil in Chile. Educ. Sci. 2020, 10, 244

30. Araya, R.; Arias Ortiz, E.; Bottan, N.; Cristia, J. Does Gamification in Education Work? Experimental Evidence from Chile. IDB WORKING PAPER SERIES № IDB-WP-982 Inter-American Development Bank. 2019

31. Honey M., Pearson G., Schweingruber H. STEM integration in K-12 education: Status, prospects, and an agenda for research. National Academies Press. 2014.

32. Araya, R. Estrategias de enseñanza de las matemáticas. Internet course for Latin American teachers of basic education, Organization of American States (OAS). 2000 http://www.educoas.org/portal/ineam/cursos_2011/MATE-E114_11.aspx?culture=es

33. Araya, R. Math Teacher Education to Prepare Students for the 21st Century Jobs: Mathematical Modeling and Computational Thinking. Submitted 